\title{
AN UNCOUNTABLE, CLOSED, AND NON-DENSE POINT SET EACH OF WHOSE COMPLE- MENTARY INTERVALS ABUTS ON ANOTHER ONE AT EACH OF ITS ENDS*
}

BY R. L. MOORE

On page 92 of the 1907 edition of Hobson's The Theory of Functions of a Real Variable, and again on page 113 of the second edition of the same treatise, there occurs the following statement: $\dagger$

" $A$ non-dense closed set is enumerable if its complementary intervals are such that every one of them abuts on another one at each of its ends."

To prove this statement, Hobson lets $G$ denote the nondense closed set in question and argues, in part, as follows:

"In this case, all the points of $G$ are either end-points of adjacent intervals, or limiting points, on both sides, of a sequence of such end-points; unless $a \ddagger$ or $b \ddagger$ be a limiting point, in which case it belongs to $G$. The end-points have the same cardinal number as the rational numbers, since the set of intervals is enumerable. Moreover the external $\S$ points form a finite set, or an enumerable set; because to each such external point there corresponds an enumerable set of endpoints of which it is the limiting point and in this correspondence any one end-point can correspond to at most two limiting points, one on each side of it."

Just what is the meaning of the above italicized statement? If $P$ is an external point, then, by definition, there exists a

* Presented to the Society, December 29, 1922.

$\dagger$ This statement will be called Proposition A.

$\ddagger$ Here $a$ and $b$ apparently denote the end-points of some interval which contains the set $G$.

$\S$ External points are defined by Hobson as points such as are not end-points of any contiguous interval but are limit points on both sides of such end-points. 
sequence of end-points (of intervals complementary to $G$ ) such that $P$ is the sequential limiting point of this sequence. But clearly there exist infinitely many such sequences and if $X$ is an end-point of any interval whatsoever complementary to $G$ then there is one sequence of such end-points which contains $X$ and has $P$ as its limiting point. In the correspondence imagined by Hobson does $P$ correspond, then, to every such point $X$ ? If not, then apparently it is intended that for each point $P$ there should be selected, in some manner or other, some particular sequence of end-points converging to $P$ and that the points of this particular sequence only should be considered as corresponding to $P$. Even if this be done, however, what justification (if any) is there for the statement that, in the correspondence so established, any one end-point can correspond to at most two limit points, one on each side of it? Certainly it is possible that for three different external points $P_{1}, P_{2}, P_{3}$ there should be selected three sequences $P_{11}, P_{12}, P_{13}, \cdots ; P_{21}, P_{22}, P_{23}, \cdots ; P_{31}, P_{32}, P_{33}, \cdots$ such that (1) these sequences converge respectively to $P_{1}, P_{2}, P_{3}$, and their points correspond respectively to the points $P_{1}, P_{2}$, $P_{3}$, and (2) $P_{11}=P_{21}=P_{31}$. In this case the end-point $P_{11}$ would correspond to three different limiting points, contrary to Hobson's statement. In fact there exists a non-dense closed point set $G$ satisfying the hypothesis of Hobson's proposition and such that there is no way whatsoever of establishing the correspondence indicated by Hobson without having some end-point correspond to uncountably many limiting points. To see this, let $K$ denote a non-dense perfect set of points lying on an interval $A B$ and containing the points $A$ and $B$. Consider the intervals $A_{1} B_{1}, A_{2} B_{2}, A_{3} B_{3}, \ldots$ which are complementary to $K$ (with respect to $A B$ ). For every two positive integers $n$ and $m$ let $P_{n m}$ and $\bar{P}_{n m}$ denote points between $A_{n}$ and $B_{n}$ situated so that $P_{n m} A_{n}=\bar{P}_{n m} B_{n}$ $=A_{n} B_{n} / 2 m$. Let $G$ denote the point set obtained by adding, to the point set $K$, all the $P_{n m}$ 's and all the $\bar{P}_{n m}$ 's.

The existence of this example disproves Proposition A.

The University of Texas 